\title{
A produção científica em Epidemiologia na Revista Ciência \& Saúde Coletiva
}

\author{
The scientific production in Epidemiology \\ of the Journal Ciência \& Saúde Coletiva
}

Antônio Augusto Moura da Silva (https://orcid.org/0000-0003-4968-5138) ${ }^{1}$

Sérgio Viana Peixoto (https://orcid.org/0000-0001-9431-2280) 2,3

\footnotetext{
${ }^{1}$ Departamento de Saúde Pública, Universidade Federal do Maranhão. R. Barão de Itapary 166, Centro. 65020-070 São Luís MA Brasil. aamouradasilva@gmail.com ${ }^{2}$ Núcleo de Estudos em Saúde Pública e Envelhecimento, Instituto René Rachou, Fiocruz. Belo Horizonte MG Brasil. ${ }^{3}$ Escola de Enfermagem, Universidade Federal de Minas Gerais. Belo Horizonte, Minas Gerais, Brasil.
}

\begin{abstract}
The aim of this article is to present a description and analysis of the scientific production in Epidemiology of the Journal Ciência \& Saúde Coletiva since its creation in 1996. The titles and abstracts of all articles published in three periods (1996 to 2002, 2011 and 2019) were read and those related to Epidemiology were analyzed. These publications were classified according to the thematic area, study design, sampling method and sample size, and place of origin of the first author. The percentage of epidemiological articles published in the journal jumped from $12.8 \%$ in the first period to $31.0 \%$ in 2011 , and to $55.3 \%$ in 2019. The most studied topics were chronic non-communicable diseases (20.3\%), nutritional epidemiology (12.2\%) and evaluation of health systems, policies, programs and services (10.9\%). Descriptive/cross-sectional studies, conducted among adults and from the Southeast region, predominated. The results indicate the need for greater participation of cohort studies, case-control and clinical trials in the scope of the journal's publications, in addition to articles that use more sophisticated statistical methods and well-defined theoretical models, which may contribute in a more relevant way to prevention and control of health problems.
\end{abstract}

Key words Epidemiology, Public health, Editorial policies, Journal articles
Resumo O objetivo deste artigo é apresentar uma descrição e análise da produção científica em Epidemiologia da Revista Ciência er Saúde Coletiva desde sua criação, em 1996. Os títulos e os resumos de todos os artigos publicados em três períodos (1996 a 2002, 2011 e 2019) foram lidos e aqueles relacionados à área da Epidemiologia foram analisados. Essas publicações foram classificadas quanto à área temática, tipo de estudo, procedimento e tamanho amostral e local de procedência do autor principal. O percentual de artigos epidemiológicos publicados na revista saltou de 12,8\% no primeiro período para 31,0\% em 2011 e 55,3\% em 2019. Os temas mais estudados foram as doenças crônicas não transmissivivis (20,3\%), epidemiologia nutricional (12,2\%) e avaliação de sistemas, políticas, programas e serviços de saúde (10,9\%). Predominaram os artigos descritivos/ transversais, conduzidos entre adultos e provenientes da região Sudeste. Os resultados indicam a necessidade de maior participação de estudos de coorte, caso-controle e ensaios clínicos no escopo das publicações da revista, além de artigos que utilizem métodos estatísticos mais sofisticados e modelos teóricos bem definidos, o que poderá contribuir de forma mais relevante para prevenção e controle de agravos à saúde.

Palavras-chave Epidemiologia, Saúde coletiva, Políticas editoriais, Artigo de revista 


\section{Introdução}

A Epidemiologia, apesar do seu intenso desenvolvimento metodológico observado nos últimos anos, ainda é uma disciplina relativamente nova em termos conceituais ${ }^{1}$. A despeito de que seus fundamentos básicos tenham se consolidado na segunda metade do século passado ${ }^{1}$, mesmo nos dias atuais ainda não se tem acordo sobre o real objeto da disciplina ${ }^{2}$ ou mesmo acerca de uma definição amplamente aceita do fenômeno do confundimento ${ }^{3}$.

Apesar disso, em meio a essa crise de identidade e do contínuo processo de construção desses conceitos, a epidemiologia brasileira floresceu. Marcos na história da disciplina no Brasil foram a formação dos primeiros epidemiologistas brasileiros em renomadas universidades no exterior $^{4}$, a criação da Abrasco (Associação Brasileira de Saúde Coletiva); da Comissão de Epidemiologia da $\mathrm{Abrasco}^{5}$; a realização dos Congressos de Epidemiologia $^{6}$; a elaboração de quatro Planos Diretores para o Desenvolvimento da Epidemiologia no Brasil'; a criação do CENEPI (Centro Nacional de Epidemiologia $)^{8}$, hoje incorporado à Secretaria de Vigilância em Saúde - SVS ${ }^{9}$; e a implantação e consolidação de programas de pósgraduação em Saúde Coletiva e Epidemiologia por todo o país ${ }^{10,11}$. Instituições como a Organização Pan-americana de Saúde (OPAS) e a Escola Nacional de Saúde Pública tiveram, também, importante participação nesse processo ${ }^{5}$.

Ao longo desse período, a produção científica brasileira em Epidemiologia apresentou crescimento vertiginoso $0^{5,12}$. Revistas mais antigas e tradicionais da área, tal como a Revista de Saúde Pública, criada em 1967, ao lado de novas revistas criadas nos anos 1980 e 1990, como os Cadernos de Saúde Pública, criado em 1985, foram responsáveis pela divulgação da maior parte da produção científica brasileira em Epidemiologia desde então ${ }^{10}$. Dentre essas, figura a Revista Ciência \& Saúde Coletiva, criada pela Abrasco, em 1996 ${ }^{13}$, que passa então a compor esse elenco de veículos para divulgação da produção científica em Epidemiologia no Brasil.

O objetivo do presente artigo é apresentar uma descrição e análise da produção científica em Epidemiologia na Revista Ciência \& Saúde Coletiva ao longo dos seus 25 anos de existência.

\section{Metodologia}

O presente estudo é de natureza descritiva, conduzido para conhecer o perfil das publicações de artigos epidemiológicos na Revista Ciência \& Saúde Coletiva ao longo do tempo. Devido à grande quantidade de artigos publicados, não foi possível abranger todo o período, sendo escolhidos os anos iniciais, um ano intermediário e outro mais recente. Não foi utilizada busca por palavras-chave, pois foi considerado que por esse método haveria dificuldade em separar artigos epidemiológicos de artigos de outras áreas do conhecimento. Dessa forma, foi realizada leitura exaustiva dos títulos e resumos dos artigos publicados na revista, por meio da base Scielo, em três períodos: 1996 a 2002 (abrangendo 15 fascículos dos volumes de 1 a 7 , num total de 196 artigos), e os anos de 2011 (correspondente ao volume 16, sendo 12 fascículos normais e um suplemento, num total de 462 artigos) e 2019 (abrangendo o volume 24 , com 12 fascículos, totalizando 425 manuscritos publicados). Os períodos foram selecionados buscando agregar, em cada um deles, um número semelhante de fascículos, de modo a não prejudicar a comparação temporal. Por esse motivo, o período inicial incluiu sete anos, totalizando 15 fascículos.

Por meio desse procedimento foram identificados artigos considerados pelos dois autores como pertencentes ao campo de conhecimento da epidemiologia. A partir da identificação de artigos epidemiológicos, foi calculado o percentual de participação desses artigos no total da produção científica publicada na Revista Ciência \& Saúde Coletiva nos três períodos analisados.

A produção científica selecionada foi categorizada de acordo com a classificação temática empregada na submissão de resumos de trabalhos para o $11^{\circ}$ Congresso Brasileiro de Epidemiologia da $\mathrm{Abrasco}^{14}$. Além disso, foram também verificadas as seguintes informações: tipo de estudo epidemiológico, grupo populacional estudado, tipo de procedimento de amostragem empregado, tamanho da amostra (codificada em $\leq 100,100$ a 499,500 a 999, 1000 a 4999 e $\geq 1000$ ) e local de trabalho do autor principal. Adicionalmente, foi realizada avaliação qualitativa dos artigos quanto aos métodos de análise estatística empregados, controle de confundimento, uso de modelos teóricos, entre outros aspectos que pudessem auxiliar a caracterização das produções avaliadas.

Os dados foram compilados e analisados utilizando os programas $\mathrm{Excel}^{\circledR}$, do pacote Microsoft 
Office, versão 2010, e Stata versão 14.0 (StataCorp LLC, College Station, TX), tendo sido apresentados em tabelas, com frequências simples e relativas.

\section{Resultados}

O percentual de artigos epidemiológicos publicados na revista Ciência \& Saúde Coletiva saltou de $12,8 \%$ no período de 1996 a 2002 para 31,0\% em 2011. Em seguida aumentou novamente para 55,3\% em 2019 (Tabela 1).

Os tópicos mais estudados foram epidemiologia das doenças crônicas não transmissíveis $(20,3 \%)$, epidemiologia nutricional (12,2\%), avaliação de sistemas, políticas, programas e serviços de saúde (10,9\%), epidemiologia da saúde bucal (10,9\%), epidemiologia da saúde do idoso $(8,4 \%)$, epidemiologia das doenças transmissíveis $(7,2 \%)$, saúde do trabalhador $(6,5 \%)$ e epidemiologia dos acidentes, violências e lesões físicas (5,5\%), com pequena variação entre 2011 e 2019. Entretanto, no primeiro período (1996 a 2002), temas relacionados à epidemiologia social e determinantes sociais em saúde $(20,0 \%)$ e artigos abordando aspectos teórico-conceituais em epidemiologia $(16,0 \%)$ foram os mais publicados. Alguns temas relevantes, como avaliação de tecnologias médico-sanitárias; ensino e formação em Epidemiologia; interdisciplinaridade e intersetorialidade na epidemiologia; sustentabilidade, integridade e ética em pesquisas epidemiológicas; uso de métodos qualitativos em estudos epidemiológicos; e vigilância epidemiológica e vigilância em saúde não tiveram participação nos três períodos avaliados (Tabela 2).

A Tabela 3 apresenta algumas características dos artigos epidemiológicos publicados em cada um dos períodos analisados. Cerca de 77\% dos estudos epidemiológicos eram descritivos ou transversais, seguidos das revisões sistemáticas

Tabela 1. Número e percentual de publicações de Epidemiologia na revista Ciência \& Saúde Coletiva, 1996-2002, 2011 e 2019.

\begin{tabular}{lcc}
\hline \multicolumn{1}{c}{ Anos } & $\begin{array}{c}\text { Total de artigos } \\
\text { publicados }^{*}\end{array}$ & $\begin{array}{c}\% \\
\text { Epidemiologia }\end{array}$ \\
\hline 1996 a 2002 & 196 & 12,8 \\
2011 & 462 & 31,0 \\
2019 & 425 & 55,3 \\
\hline
\end{tabular}

(5,5\%), dos estudos ecológicos $(4,7 \%)$ e com pequenas flutuações nos dois momentos mais recentes. Considerando os três períodos analisados, estudos conduzidos entre adultos $(22,5 \%)$, todas as faixas etárias $(18,8 \%)$ e idosos $(17,8 \%)$ foram mais frequentes do que aqueles que incluíram a população materno-infantil $(15,7 \%)$ e adolescentes $(9,7 \%)$. Amostras probabilísticas de base populacional $(35,3 \%)$ e amostras intencionais ou de conveniência $(33,6 \%)$ predominaram, enquanto as amostras probabilísticas de população restrita $(15,8 \%)$ e estudos abrangendo o universo $(15,3 \%)$ foram menos frequentes. Estudos com tamanhos amostrais de 100 a 499 indivíduos foram mais frequentes $(38,6 \%)$, mas estudos com grande tamanho amostral $(\geq 1.000)$ também tiveram boa representação. Em relação a esse aspecto, algumas variações foram observadas ao longo dos três períodos investigados, chamando atenção para a predominância de artigos que utilizaram amostras probabilísticas de base populacional e amostras grandes $(\geq 5.000)$ no primeiro período investigado (Tabela 3 ).

Em relação à distribuição regional dos autores principais dos artigos (Tabela 4), 46,4\% vieram do Sudeste, $20,6 \%$ do Sul, 18,9\% do Nordeste, $6,0 \%$ do Centro-Oeste, 6,5\% do exterior e apenas $1,7 \%$ da região Norte. No primeiro período, o Sudeste concentrava $80,0 \%$ dos autores, mas sua participação diminuiu para menos de $50 \%$ em 2011 e 2019. A participação de artigos de autores do exterior foi mais elevada no último período, quando alcançou 8,5\% do total (Tabela 4).

Os estudos transversais na sua maioria objetivaram estimar prevalência e estudar fatores de risco para uma determinada condição e alguns respondiam a uma pergunta mais específica, com uma exposição e um desfecho. De modo geral, os métodos utilizados foram os da epidemiologia dos fatores de risco, tais como frequências relativas, cálculo das razões de risco, razões de prevalência ou odds ratio em modelos multivariáveis, geralmente regressão logística ou de Poisson com ajuste robusto da variância. A estratégia utilizada para controle do confundimento raramente foi mencionada. No entanto, o método mais utilizado foi baseado em $\mathrm{p}$ valores, muitas vezes realizado de forma automatizada (stepwise). A imensa maioria dos estudos não tinha um modelo teórico mais elaborado subjacente que auxiliasse na interpretação dos achados. Dentre os poucos modelos teóricos utilizados o mais frequente foi a modelagem hierarquizada. Análises estatísticas mais sofisticadas, como modelos multiníveis ou análise com escore de propensão, foram rara- 
Tabela 2. Classificação temática dos artigos epidemiológicos publicados na revista Ciência \& Saúde Coletiva, 1996-2002, 2011 e 2019.

\begin{tabular}{|c|c|c|c|c|c|c|c|c|}
\hline \multirow{2}{*}{ Temática } & \multicolumn{2}{|c|}{1996 a 2002} & \multicolumn{2}{|c|}{2011} & \multicolumn{2}{|c|}{2019} & \multicolumn{2}{|c|}{ Total } \\
\hline & $\mathbf{n}$ & $\%$ & $\mathbf{n}$ & $\%$ & $\mathbf{n}$ & $\%$ & $\mathbf{n}$ & $\%$ \\
\hline Aspectos teórico-conceituais em epidemiologia & 4 & 16,0 & - & - & - & - & 4 & 1,0 \\
\hline $\begin{array}{l}\text { Avaliação de sistemas, políticas, programas e serviços de } \\
\text { saúde }\end{array}$ & 3 & 12,0 & 23 & 16,1 & 18 & 7,7 & 44 & 10,9 \\
\hline Avaliação de tecnologias médico-sanitárias & - & - & - & - & - & - & - & - \\
\hline Ensino e formação em epidemiologia & - & - & - & - & - & - & - & - \\
\hline Epidemiologia da saúde bucal & - & - & 14 & 9,8 & 22 & 9,4 & 36 & 8,9 \\
\hline Epidemiologia da saúde da criança & - & - & 4 & 2,8 & 5 & 2,1 & 9 & 2,2 \\
\hline Epidemiologia da saúde do adolescente & - & - & 3 & 2,1 & 2 & 0,9 & 5 & 1,2 \\
\hline Epidemiologia da saúde do adulto & - & - & 1 & 0,7 & - & - & 1 & 0,2 \\
\hline Epidemiologia da saúde do idoso & 2 & 8,0 & 8 & 5,6 & 24 & 10,2 & 34 & 8,4 \\
\hline $\begin{array}{l}\text { Epidemiologia das doenças crônicas não-transmissíveis } \\
\text { (DCNT) }\end{array}$ & 3 & 12,0 & 30 & 21,0 & 49 & 20,9 & 82 & 20,3 \\
\hline Epidemiologia das doenças transmissíveis & 3 & 12,0 & 13 & 9,1 & 13 & 5,5 & 29 & 7,2 \\
\hline Epidemiologia do curso de vida & - & - & 1 & 0,7 & - & - & 1 & 0,2 \\
\hline Epidemiologia dos acidentes, violências e lesões físicas & 1 & 4,0 & 3 & 2,1 & 18 & 7,7 & 22 & 5,5 \\
\hline Epidemiologia em subgrupos populacionais específicos & - & - & - & - & 8 & 3,4 & 8 & 2,0 \\
\hline Epidemiologia genética e molecular & 2 & 8,0 & - & - & - & - & 2 & 0,5 \\
\hline Epidemiologia nutricional & - & - & 14 & 9,8 & 35 & 14,9 & 49 & 12,2 \\
\hline Epidemiologia social e determinantes sociais em saúde & 5 & 20,0 & 2 & 1,4 & 7 & 3,0 & 14 & 3,5 \\
\hline Farmacoepidemiologia & - & - & 6 & 4,2 & 4 & 1,7 & 10 & 2,5 \\
\hline Interdisciplinaridade e intersetorialidade na epidemiologia & - & - & - & - & - & - & - & - \\
\hline Métodos e técnicas em estudos epidemiológicos & - & - & 4 & 2,8 & 1 & 0,4 & 5 & 1,2 \\
\hline Outros tópicos em epidemiologia & - & - & 1 & 0,7 & 2 & 0,9 & 3 & 0,7 \\
\hline Saúde ambiental e desastres & 1 & 4,0 & 4 & 2,8 & 6 & 2,6 & 11 & 2,7 \\
\hline Saúde do trabalhador & - & - & 8 & 5,6 & 18 & 7,7 & 26 & 6,5 \\
\hline Sistemas de informação em saúde & 1 & 4,0 & 4 & 2,8 & 3 & 1,3 & 8 & 2,0 \\
\hline $\begin{array}{l}\text { Sustentabilidade, integridade e ética em pesquisas } \\
\text { epidemiológicas }\end{array}$ & - & - & - & - & - & - & - & - \\
\hline Uso de métodos qualitativos em estudos epidemiológicos & - & - & - & - & - & - & - & - \\
\hline Vigilância epidemiológica e vigilância em saúde & - & - & - & - & - & - & - & - \\
\hline
\end{tabular}

mente utilizadas. Nos períodos estudados não foi publicado artigo utilizando DAGs (gráficos acíclicos direcionados), abordagem contrafatual, modelagem com equações estruturais, análises com big data ou modelos bayesianos (dados não mostrados em tabelas).

\section{Discussão}

A Epidemiologia é o campo que concentra atualmente a maior parte das publicações da Revista Ciência \& Saúde Coletiva, tal como já ocorre com outras revistas de Saúde Pública brasileiras, como os Cadernos de Saúde Pública ${ }^{15}$ e a Revista de Saúde Pública ${ }^{5}$. Entretanto, chama atenção que apesar da grande participação de revistas nacionais, parte relevante da produção epidemiológica brasileira, especialmente a de melhor qualidade, se concentra em periódicos internacionais ${ }^{5}$.

No século passado, predominava na produção epidemiológica latino-americana a temática das doenças transmissíveis e da saúde materno -infantil ${ }^{5,10,16}$. Talvez por se tratar de uma revista mais nova, a produção epidemiológica predominante na Ciência \& Saúde Coletiva é de doenças crônicas não transmissíveis, de forma semelhante ao observado em revistas de países de alta renda ${ }^{17}$. Essa maior produção sobre a temática de doenças e agravos não transmissíveis reflete a maior carga global de doenças desses grupos de causas para a população brasileira ${ }^{18}$, demonstrando um ali- 
Tabela 3. Tipos de estudos epidemiológicos, população, amostragem e tamanho da amostra dos artigos epidemiológicos publicados na revista Ciência \& Saúde Coletiva, 1996-2002, 2011 e 2019.

\begin{tabular}{|c|c|c|c|c|c|c|c|c|}
\hline \multirow{2}{*}{ Variáveis } & \multicolumn{2}{|c|}{1996 a 2002} & \multicolumn{2}{|c|}{2011} & \multicolumn{2}{|c|}{2019} & \multicolumn{2}{|c|}{ Total } \\
\hline & $\mathbf{n}$ & $\%$ & $\mathbf{n}$ & $\%$ & $\mathbf{n}$ & $\%$ & $\mathbf{n}$ & $\%$ \\
\hline \multicolumn{9}{|l|}{ Tipo de estudo } \\
\hline Descritivo/Transversal & 18 & 72,0 & 114 & 79,7 & 179 & 76,2 & 311 & 77,2 \\
\hline Coorte & - & - & 2 & 1,4 & 11 & 4,7 & 13 & 3,2 \\
\hline Caso controle & - & - & 1 & 0,7 & 6 & 2,6 & 7 & 1,7 \\
\hline Ecológico & - & - & 6 & 4,2 & 13 & 5,5 & 19 & 4,7 \\
\hline Tendências em séries temporais & - & - & 6 & 4,2 & 5 & 2,1 & 11 & 2,7 \\
\hline Análise espacial & - & - & 1 & 0,7 & 1 & 0,4 & 2 & 0,5 \\
\hline Revisão sistemática & - & - & 6 & 4,2 & 16 & 6,8 & 22 & 5,5 \\
\hline Experimental pré e pós & - & - & 3 & 2,1 & 2 & 0,9 & 5 & 1,2 \\
\hline Ensaio clínico & - & - & - & - & 1 & 0,4 & 1 & 0,2 \\
\hline Outros & 7 & 28,0 & 4 & 2,8 & 1 & 0,4 & 12 & 3,0 \\
\hline \multicolumn{9}{|l|}{ População* } \\
\hline Materno-infantil & - & - & 16 & 11,8 & 44 & 19,2 & 60 & 15,7 \\
\hline Adolescente & - & - & 13 & 9,6 & 24 & 10,5 & 37 & 9,7 \\
\hline Adulto & 1 & 5,6 & 40 & 29,4 & 45 & 19,7 & 86 & 22,5 \\
\hline Idoso & 2 & 11,1 & 12 & 8,8 & 54 & 23,6 & 68 & 17,8 \\
\hline Adulto e idoso & 4 & 22,2 & 1 & 0,7 & 19 & 8,3 & 24 & 6,3 \\
\hline Todos & 9 & 50,0 & 35 & 25,7 & 28 & 12,2 & 72 & 18,8 \\
\hline Criança e adolescente & - & - & 8 & 5,9 & 7 & 3,1 & 15 & 3,9 \\
\hline Mulher & 2 & 11,1 & 11 & 8,1 & 8 & 3,5 & 21 & 5,5 \\
\hline \multicolumn{9}{|l|}{ Amostragem ${ }^{\star}$} \\
\hline Intencional & 1 & 5,6 & 40 & 29,4 & 80 & 38,8 & 121 & 33,6 \\
\hline Probabilística de base populacional & 13 & 72,2 & 39 & 28,7 & 75 & 36,4 & 127 & 35,3 \\
\hline Probabilística de população restrita & 1 & 5,6 & 30 & 22,1 & 26 & 12,6 & 57 & 15,8 \\
\hline Universo & 3 & 16,7 & 27 & 19,9 & 25 & 12,1 & 55 & 15,3 \\
\hline \multicolumn{9}{|l|}{ Tamanho da amostra ${ }^{\star}$} \\
\hline$<100$ & 1 & 6,7 & 12 & 9,8 & 22 & 10,9 & 35 & 10,3 \\
\hline 100 a 499 & 1 & 6,7 & 60 & 48,8 & 70 & 34,8 & 131 & 38,6 \\
\hline 500 a 999 & - & - & 19 & 15,4 & 42 & 20,9 & 61 & 18,0 \\
\hline 1000 a 4999 & 2 & 13,3 & 16 & 13,0 & 34 & 16,9 & 52 & 15,3 \\
\hline$\geq 5000$ & 11 & 73,3 & 16 & 13,0 & 33 & 16,4 & 60 & 17,7 \\
\hline
\end{tabular}

* Os números são menores que os totais de artigos avaliados em cada ano, pois os artigos de revisão sistemática e aspectos teóricos e metodológicos não foram classificados quanto a essas características.

nhamento entre a produção da área com o perfil de saúde da população.

De maneira geral, nota-se grande diversidade das temáticas abordadas nas investigações dos epidemiologistas brasileiros ${ }^{12}$, como observado nas produções da Revista Ciência \& Saúde Coletiva. Entre 2011 e 2019, houve crescimento da área de Epidemiologia Nutricional, atingindo 14,9\% nesse último ano, o que já tinha sido descrito anteriormente $e^{5}$. Outras áreas que se destacam são epidemiologia da saúde bucal, epidemiologia dos acidentes, violências e lesões físicas, epidemiologia das doenças transmissíveis e epidemiologia da saúde do idoso. Ressalta-se ainda a importante presença da área de avaliação de serviços, políticas e programas de saúde, demonstrando a forte interação entre a pesquisa acadêmica e as práticas dos serviços de saúde, que é considerada uma das características definidoras da epidemiologia brasileira ${ }^{19}$. Como tendências negativas destaca-se o virtual desaparecimento de estudos abrangendo aspectos teórico-conceituais da epidemiologia e grande redução no percentual de publicações nas temáticas Epidemiologia Social e determinantes sociais em Saúde. Além disso, interessante notar que houve apenas uma publicação sobre 
Tabela 4. Local da instituição de vínculo do autor principal dos artigos epidemiológicos publicados na revista Ciência \& Saúde Coletiva, 1996-2002, 2011 e 2019.

\begin{tabular}{|c|c|c|c|c|c|c|c|c|}
\hline \multirow{2}{*}{ Regiões e Estados } & \multicolumn{2}{|c|}{1996 a 2002} & \multicolumn{2}{|c|}{2011} & \multicolumn{2}{|c|}{2019} & \multicolumn{2}{|c|}{ Total } \\
\hline & $\mathbf{n}$ & $\%$ & $\mathbf{n}$ & $\%$ & $\mathbf{n}$ & $\%$ & $\mathbf{n}$ & $\%$ \\
\hline Região Norte & - & - & 4 & 2,8 & 3 & 1,3 & 7 & 1,7 \\
\hline Rondônia & - & - & - & - & - & - & - & - \\
\hline Acre & - & - & - & - & 1 & 0,4 & 1 & 0,2 \\
\hline Amazonas & - & - & 4 & 2,8 & 1 & 0,4 & 5 & 1,2 \\
\hline Roraima & - & - & - & - & - & - & - & - \\
\hline Pará & - & - & - & - & - & - & - & - \\
\hline Amapá & - & - & - & - & - & - & - & - \\
\hline Tocantins & - & - & - & - & 1 & 1 & 1 & 0 \\
\hline Região Nordeste & 1 & 4,0 & 24 & 16,8 & 51 & 21,7 & 76 & 18,9 \\
\hline Maranhão & - & - & 2 & 1,4 & 4 & 1,7 & 6 & 1,5 \\
\hline Piauí & - & - & 1 & 0,7 & 2 & 0,9 & 3 & 0,7 \\
\hline Ceará & - & - & 4 & 2,8 & 6 & 2,6 & 10 & 2,5 \\
\hline Rio Grande do Norte & - & - & 3 & 2,1 & 5 & 2,1 & 8 & 2,0 \\
\hline Paraíba & - & - & - & - & 8 & 3,4 & 8 & 2,0 \\
\hline Pernambuco & - & - & 9 & 6,3 & 11 & 4,7 & 20 & 5,0 \\
\hline Alagoas & - & - & 2 & 1,4 & 2 & 0,9 & 4 & 1,0 \\
\hline Sergipe & - & - & 1 & 0,7 & 1 & 0,4 & 2 & 0,5 \\
\hline Bahia & 1 & 4,0 & 2 & 1,4 & 12 & 5,1 & 15 & 3,7 \\
\hline Região Sudeste & 20 & 80,0 & 64 & 44,8 & 103 & 43,8 & 187 & 46,4 \\
\hline Minas Gerais & 4 & 16,0 & 21 & 14,7 & 46 & 19,6 & 71 & 17,6 \\
\hline Espírito Santo & - & - & 3 & 2,1 & 8 & 3,4 & 11 & 2,7 \\
\hline Rio de Janeiro & 13 & 52,0 & 12 & 8,4 & 19 & 8,1 & 44 & 10,9 \\
\hline São Paulo & 3 & 12,0 & 28 & 19,6 & 30 & 12,8 & 61 & 15,1 \\
\hline Região Sul & 2 & 8,0 & 36 & 25,2 & 45 & 19,1 & 83 & 20,6 \\
\hline Paraná & & & 9 & 6,3 & 11 & 4,7 & 20 & 5,0 \\
\hline Santa Catarina & & & 8 & 5,6 & 8 & 3,4 & 16 & 4,0 \\
\hline Rio Grande do Sul & 2 & 8,0 & 19 & 13,3 & 26 & 11,1 & 47 & 11,7 \\
\hline Região Centro-Oeste & - & - & 11 & 7,7 & 13 & 5,5 & 24 & 6,0 \\
\hline Mato Grosso do Sul & - & - & 4 & 2,8 & 3 & 1,3 & 7 & 1,7 \\
\hline Mato Grosso & - & - & - & - & 2 & 0,9 & 2 & 0,5 \\
\hline Goiás & - & - & - & - & 2 & 0,9 & 2 & 0,5 \\
\hline Distrito Federal & - & - & 7 & 4,9 & 6 & 2,6 & 13 & 3,2 \\
\hline Exterior & 2 & 8,0 & 4 & 2,8 & 20 & 8,5 & 26 & 6,5 \\
\hline Portugal & - & - & - & - & 7 & 3,0 & 7 & 1,7 \\
\hline Espanha & - & - & - & - & 4 & 1,7 & 4 & 1,0 \\
\hline Estados Unidos & 2 & 8,0 & 1 & 0,7 & - & - & 3 & 0,7 \\
\hline Canadá & - & - & 1 & 0,7 & - & - & 1 & 0,2 \\
\hline México & - & - & 2 & 1,4 & 4 & 1,7 & 6 & 1,5 \\
\hline Chile & - & - & - & - & 1 & 0,4 & 1 & 0,2 \\
\hline Colômbia & - & - & - & - & 1 & 0,4 & 1 & 0,2 \\
\hline Turquia & - & - & - & - & 3 & 1,3 & 3 & 0,7 \\
\hline
\end{tabular}

epidemiologia do curso de vida, em 2011, que é uma temática emergente na área ${ }^{6}$. Outro ponto digno de nota é que várias revisões sistemáticas publicadas não seguiram as recomendações do
PRISMA (Preferred Reporting Items for Systematic Reviews and Meta-Analyses ${ }^{20}$, refletindo a diversidade de terminologias e de compreensão do que sejam revisões de literatura ${ }^{21}$. 
Em contraste com outras revistas epidemiológicas internacionais, onde o tipo de estudo predominante é o de coorte ${ }^{17}$, dentre os artigos epidemiológicos publicados na Revista Ciência \& Saúde Coletiva predominaram os descritivos/ transversais, que geralmente estudam vários fatores de risco para um determinado desfecho, sendo, em sua maioria, de natureza exploratória. Dentre os fatores de risco, geralmente maior ênfase é colocada naqueles ligados ao estilo de vida do que a determinantes estruturais e socioeconômicos das doenças. O estudo simultâneo de vários fatores de risco, geralmente realizado sem uma teoria subjacente que lhe dê suporte, pode induzir a erro na interpretação do efeito de variáveis mais distais. Em muitas situações, o efeito direto de uma variável distal é interpretado como representando o efeito total, o que conduz à falsa conclusão de que a variável não estaria associada ao desfecho. Esse problema foi bem analisado e denominado "a falácia da tabela 2 " em estudo anterior $^{22}$.

Análise qualitativa dos artigos publicados na Revista Ciência \& Saúde Coletiva mostra que há pouco uso de modelos teóricos que auxiliem na interpretação dos dados e esses, quando usados, são bastante simples. Da mesma forma, geralmente não é explicitado como foram selecionadas as variáveis de ajuste para confundimento e, nessa seleção, maior ênfase é dada a pontos de corte baseados na significância do que em uma teoria subjacente. Todas essas características não são exclusivas dos artigos publicados na Revista Ciência \& Saúde Coletiva. Em editorial de grande repercussão, publicado em 2013, as editoras dos Cadernos de Saúde Pública descreveram essas questões naquela revista e denominaram esse círculo vicioso de "mais do mesmo"15. Motivos para essa falta de criatividade e uso de formas automatizadas de fazer ciência podem ser parcialmente atribuídas à pressão de publicar para ser bem sucedido na carreira acadêmica, o fenômeno denominado "publish or perish"15. No Brasil essa situação foi provavelmente potencializada pelo modelo de avaliação da pós-graduação, hoje em revisão, que atribuía maior importância para a quantidade de produtos, em detrimento da sua qualidade $^{23}$. Isso talvez seja um dos fatores responsáveis pelo fato da produção brasileira em Saúde Coletiva ter pouca visibilidade internacio$\mathrm{nal}^{23}$.

Sobre a distribuição regional das publicações, nota-se nos dois primeiros períodos, forte concentração da produção no Sudeste, especialmente em Minas Gerais, São Paulo e Rio de Janeiro, como já foi descrito anteriormente ${ }^{10,13}$. No último período, houve importante desconcentração geográfica da produção epidemiológica observada na Ciência \& Saúde Coletiva, com aumento da participação de autores da região Nordeste e Sul, embora ainda predominem artigos de autores vinculados a instituições do Sudeste. Essa distribuição das publicações é coerente com a distribuição regional dos Programas de Pós-Graduação da área da Saúde Coletiva no Brasil, que apesar da grande expansão observada nos últimos anos, ainda está concentrada na região $\mathrm{Su}$ deste, com cerca de $45 \%$ do total de programas, enquanto o Norte e Centro-Oeste concentram apenas $11 \%$ desse total ${ }^{24}$. Quando comparado ao percentual de publicações oriundas de fora do país, há clara supremacia das produções de autores brasileiros, concentrando $94 \%$ dos artigos, o que é consonante com a tendência observada nas revistas internacionais de epidemiologia, que apresenta predomínio de publicações do próprio país de origem dos autores ${ }^{25}$.

Atualmente a taxa de recusas na revista $\mathrm{Ci}$ ência \& Saúde Coletiva é bem elevada, girando em torno de 90\%. Na área de Epidemiologia essa elevada taxa se deve a vários fatores: artigos descritivos com pequeno tamanho de amostra e/ou baseados em amostras de conveniência; estudos transversais respondendo perguntas há muito elucidadas; análises com pouco componente teórico; e discussões muito superficiais, mais comparando do que interpretando os dados. A falta de criatividade produz artigos contendo "mais do mesmo"15, com pouca contribuição relevante para o avanço do conhecimento. Além disso, o grande número de submissões obriga o corpo editorial a realizar seleção com base na prioridade, comparando artigos e escolhendo os mais promissores.

A interpretação dos resultados apresentados neste trabalho deve considerar a análise de apenas três períodos, ao longo dos 25 anos da revista, devido à dificuldade de avaliar de forma adequada toda a produção do periódico, que somou 4.928 artigos entre 1996 e 2019. Dessa forma, algumas informações descritas nesta publicação, como temáticas abordadas nos artigos, tipo de estudo e distribuição regional, não representam a totalidade da produção da Revista Ciência \& Saúde Coletiva. No entanto, cabe ressaltar que os períodos foram escolhidos de modo a representar o perfil das publicações da Revista nos anos iniciais, no ano intermediário e no ano mais recente de sua existência, possibilitando análise detalhada do material consultado em cada período e a compa- 
ração temporal desse perfil. Além disso, os resultados mostram tendências semelhantes às descritas para outros periódicos nacionais e internacionais, de constante aumento da produção científica na área da Epidemiologia ${ }^{5,12,17}$, demonstrando a possível representatividade dessa evolução.

$\mathrm{O}$ que os editores visualizam como o futuro da Epidemiologia na revista Ciência \& Saúde Coletiva? Seria importante aumentar a realização de estudos de coorte, caso-controle e ensaios clínicos controlados randomizados, focados em uma exposição por vez, ao invés de estudar vários fatores de risco simultaneamente. O uso de métodos estatísticos mais sofisticados e apropriados a responder perguntas novas são bem vindos. Artigos que partam de modelos teóricos bem estabelecidos e que tenham a análise norteada mais pela teoria e menos pela significância estatística produzirão respostas de melhor qualidade e com maior poder de influência na prevenção e controle de agravos à saúde, finalidade precípua da epidemiologia.

\section{Colaboradores}

AAM Silva e SV Peixoto: concepção do estudo, extração, análise e interpretação dos dados, redação e revisão do manuscrito, e aprovação da versão final.

\section{Agradecimentos}

AAM Silva e SV Peixoto são bolsistas de produtividade do Conselho Nacional de Desenvolvimento Científico e Tecnológico (CNPq). 


\section{Referências}

1. Rothman KJ, Greenland S, Lash TL. Modern Epidemiology. $3^{\text {rd }}$. ed. Philadelphia: Lipincott Williams \& Wilkins; 2008

2. Goldberg M. Cet obscur objet de l'épidémiologie. Sciences sociales et santé 1982; 1(1):55-110.

3. Hernán MA, Hernández-Díaz S, Werler MM, Mitchell AA. Causal Knowledge as a Prerequisite for Confounding Evaluation: An Application to Birth Defects Epidemiology. Am J Epidemiol 2002; 155(2):176-184.

4. Medronho R, Almeida-Filho N, Scliar M. Notas sobre a história da epidemiologia no Brasil. In: Almeida-Filho N, Barreto ML, organizadores. Epidemiologia \& Saúde: fundamentos, métodos, aplicações. Rio de Janeiro: Guanabara Koogan; 2012. p. 24-28.

5. Turci SRB, Guilam MCR, Câmara MCC. Epidemiologia e Saúde Coletiva: Tendências da produção epidemiológica brasileira quanto ao volume, indexação e áreas de investigação - 2001 a 2006. Cien Saude Colet 2010; 15(4):1967-1976.

6. Almeida-Filho N, Medronho R, Barreto M. Estado da arte em Epidemiologia no Brasil. In: Paim JS, Almeida-Filho N, organizadores. Saúde Coletiva: teoria e prática. Rio de Janeiro: Medbook; 2014. p. 557-565.

7. Associação Brasileira de Saúde Coletiva (Abrasco). IV Plano Diretor para o Desenvolvimento da Epidemiologia no Brasil. Rev Bras Epidemiol 2005; 8(Supl. 1):1-43.

8. Barata RB, Barreto ML. Algumas Questões sobre o Desenvolvimento da Epidemiologia na América Latina. Cien Saude Colet 1996; 1(1):70-79.

9. Teixeira MG, Costa MCN, Carmo EH, Oliveira WK, Penna GO. Health surveillance at the SUS: Development, effects and perspectives. Cien Saude Colet 2018; 23(6):1811-1818.

10. Guimarães R, Lourenço R, Cosac S. A pesquisa em epidemiologia no Brasil. Rev Saude Publica 2001; 35(4):321-340.

11. Barros AJD. Produção científica em saúde coletiva: perfil dos periódicos e avaliação pela Capes. Rev Saude Publica 2006; 40(n. esp.):43-49.

12. Barreto ML. Crescimento e tendência da produção científica em epidemiologia no Brasil. Rev Saude Publica 2006; 40(n. esp.):79-85.

13. Viacava F. Produção científica dos cursos de pósgraduação em Saúde Coletiva no período 1998-2006 Scientific production of Brazilian graduate courses in Public Health during 1998-2006. Cien Saude Colet 2010; 15(4):1977-1988.

14. Associação Brasileira de Saúde Coletiva (Abrasco). $11^{\circ}$ Congresso Brasileiro de Epidemiologia. Trabalhos. Regras Gerais para Submissão de Resumos de Trabalhos. 2020 [acessado 2020 Maio 21]. Disponível em: https://epi.org.br/trabalhos/index.php.

15. Carvalho MS, Travassos C, Coeli CM. Mais do mesmo? Cad Saude Publica 2013; 29(11):2141-2141.
16. Pellegrini-Filho A, Goldbaum M, Silvi J. Produccion de articulos cientificos sobre salud en seis paises de America Latina, 1973 a 1992. Rev Panam Salud Publica 1997; 1(1):23-34.

17. Grant C, Williams B, Driscoll T. Historical trends in publications in the International Journal of Epidemiology. Int J Epidemiol 2018; 47(3):938-941.

18. GBD 2016 Brazil Collaborators. Burden of disease in Brazil, 1990-2016: a systematic subnational analysis for the Global Burden of Disease Study 2016. Lancet 2018; 392(10149):760-775.

19. Barreto ML. Papel da epidemiologia no desenvolvimento do Sistema Único de Saúde no Brasil: histórico, fundamentos e perspectivas. Rev Bras Epidemiol 2002, 5(Supl. 1):4-17.

20. Moher D, Liberati A, Tetzlaff J, Altman DG, PRISMA Group. Preferred reporting items for systematic reviews and meta-analyses: The PRISMA statement. BMJ 2009; 339(7716):332-336.

21. Grant MJ, Booth A. A typology of reviews: An analysis of 14 review types and associated methodologies. Health Info Libr J 2009; 26(2):91-108.

22. Westreich D, Greenland S. The table 2 fallacy: presenting and interpreting confounder and modifier coefficients. Am J Epidemiol 2013; 177(4):292-298.

23. Meneguini R. Visibilidade internacional da produção brasileira em saúde coletiva. Cad Saude Publica 2010 26(6):1058-1059.

24. Brasil. Ministério da Educação (MEC). Coordenação de Aperfeiçoamento de Pessoal de Nível Superior. Diretoria de Avaliação. Documento de Área. Área 22: Saúde Coletiva, 2019 [acessado 2020 Maio 27]. Disponível em: https://www.capes.gov.br/images/Documento_de_área_2019/SAUDE_COLETIVA.pdf

25. Jacobsen KH. Patterns of co-authorship in international epidemiology. J Epidemiol Community Health 2009; 63(8):665-669.

Artigo apresentado em 04/06/2020

Aprovado em 04/06/2020

Versão final apresentada em 06/06/2020 
